\title{
Automated Real-Time Surveillance Using Health Indicator Data Received at Different Time Intervals
}

\author{
Joseph Lombardo*1, Julie Pavlin², Christopher Cuellar ${ }^{1}$, Yevgeniy Elbert ${ }^{1}$ and Jean-Paul \\ Chretien ${ }^{2}$
}

${ }^{1}$ Johns Hopkins University Applied Physics Laboratory, Laurel, MD, USA; ${ }^{2}$ Armed Forces Health Surveillance Center, Silver Spring, MD, USA

\begin{abstract}
Objective
To develop a real-time surveillance capability that processes, fuses and assesses when data is received using a new fusion processing methodology and multiple sources health indicator data.
\end{abstract}

\section{Introduction}

The Johns Hopkins Applied Physics Laboratory and the Armed Forces Health Surveillance Center have developed a hybrid processing engine that alerts monitors when a severe health condition exists based on corroboration among several sources of data. The system was designed to ingest a day's worth of recent data and provide results to monitors daily. In some theaters, the health of the US Forces must be determined at near-real time rates requiring a reassessment of current surveillance practices. Challenges exist in both acquiring data in real-time and in modifying automated alerting processes to re-evaluate as a new piece of evidence is received.

\section{Methods}

DoD surveillance uses several sources of health indicator data. It is possible that some of the data elements like reason for visit (chief complaint), ICD-9/10 or requests for laboratory tests could be made available in near real-time, while other data sources may only be available daily or weekly. Current processing techniques used in surveillance alerting look at the expected number of counts for each data source per day, but do not take into account the time of day when data is received. Many clinics are open for assigned hours while others may be open 24/7. In order to alert for abnormalities an automated system must now take into account normal variations within the day as well as the day of week.

\section{Results}

The concept will be presented since development has not begun yet.

\section{Conclusions}

The fusion processor developed for AFHSC uses a hybrid approach using statistical alerting on individual data sources followed by belief networks to find corroboration among data sources. Given that different data sources may vary widely in their time of day occurrence, the hybrid approach may lend itself to processing multiple sources of evidence all with different daily and weekly characteristics.

\section{Keywords}

Real-time processing; Data Fusion; Surveillance

\section{Acknowledgments}

This work is funded by the Armed Forces Health Surveillance Center

*Joseph Lombardo

E-mail: joe.lombardo@jhuapl.edu 